\title{
Médard, Henri \& Doyle, Shane (eds). - Slavery in the Great Lakes Region of East Africa
}

\section{Catherine Coquery-Vidrovitch}

\section{(2) OpenEdition}

1 Journals

Édition électronique

URL : http://journals.openedition.org/etudesafricaines/14124

DOI : 10.4000/etudesafricaines. 14124

ISSN : 1777-5353

Éditeur

Éditions de l'EHESS

\section{Édition imprimée}

Date de publication : 30 mars 2010

Pagination : 350-354

ISBN : 978-2-7132-2251-1

ISSN : 0008-0055

Référence électronique

Catherine Coquery-Vidrovitch, « Médard, Henri \& Doyle, Shane (eds). - Slavery in the Great Lakes Region of East Africa ", Cahiers d'études africaines [En ligne], 197 | 2010, mis en ligne le 21 avril 2010, consulté le 24 septembre 2020. URL : http://journals.openedition.org/etudesafricaines/14124 ; DOI : https:// doi.org/10.4000/etudesafricaines. 14124

Ce document a été généré automatiquement le 24 septembre 2020.

(c) Cahiers d'Études africaines 


\title{
Médard, Henri \& Doyle, Shane (eds). - Slavery in the Great Lakes Region of East Africa
}

\author{
Catherine Coquery-Vidrovitch
}

\section{RÉFÉRENCE}

MÉDARD, Henri \& DOYLE, Shane (eds). - Slavery in the Great Lakes Region of East Africa.

Oxford, James Currey (« Eastern African Studies »); Kampala, Fountain Publishers;

Nairobi, EAEP; Athens, Ohio University Press, 2007, 273 p., ill., cartes, bibl., index.

1 Voici un ouvrage collectif, résultat de recherches concertées qui ont abouti en 2002 à Paris à un colloque dont est issue la publication. il est dommage que la très solide introduction d'Henri Médard n'ait pas été publiée conjointement en français, comme cela se faisait parfois naguère. Car le sujet était resté étonnamment vierge : celui de l'existence et de l'organisation de l'esclavage interne aux sociétés africaines dans ce cœur de l'Afrique. On possédait déjà de bonnes connaissances des réseaux de traite négrière atlantique ou arabe, mais l'opinion la plus courante était que la plupart des sociétés locales les subissaient plutôt que d'y participer. Or l'ensemble des travaux présentés ici suggèrent au contraire, comme l'annonce Médard en introduction, que «l'esclavage a été beaucoup plus important dans la région que ce qu'on a souvent affirmé, et que le rôle des Africains de l'intérieur, non côtiers, dans la traite négrière a été bien plus complexe et important que ce qu'on avait coutume de penser $\aleph^{1}$. L'hypothèse interpellante est que : «La traite négrière internationale s'est développée d'abord parce que l'esclavage était déjà très répandu dans la région » (p. 32).

Bien entendu, il faut d'abord définir ce qu'on entend par le terme « esclavage ", qui ne désigne pas nécessairement un système aussi typé que celui mis en place au sud des États-Unis dans les plantations de coton. Des travaux des auteurs se dégage néanmoins une définition commune assez claire : l'esclave est un «étranger sans racine » (kinless 
outsider), devenu dépendant non libre (un-free dependent), étant entendu que tous les étrangers ne sont pas nécessairement esclaves, et que la condition libre n'est pas aisée à définir clairement dans beaucoup de sociétés anciennes africaines - la liberté étant comprise comme le respect commun de privilèges spécifiques dont jouissait un groupe ou un individu, privilèges hérités du fait du genre, de l'occupation, et surtout de la famille, du lignage ou du clan (pp. 25-27). Les auteurs s'accordent pour montrer que la condition servile était majoritairement féminine, et qu'il était beaucoup plus difficile pour une femme que pour un homme de s'en émanciper. Le sujet est spécifiquement développé par Michael W. Tuck sur le cas ougandais. Mais même pour l'homme, être libéré n'en faisait pas moins un esclave social dans la mesure où il restait dépourvu d'ancêtres. L'analyse de l'évolution plausible de l'esclavage interne depuis le Xvi siècle est surtout développée à propos du Buganda, compte tenu de l'abondance relative des sources sur ce royaume (pour la moitié des dix cas étudiés). Richard Reid relie clairement l'importance numérique des esclaves à l'édification des royaumes, qui se sont toujours constitués aux dépens de leurs voisins. Ces luttes intestines ont été pourvoyeuses de prisonniers de guerre (et de femmes) incorporés en qualité d'esclaves à la société victorieuse. Par ailleurs, contrairement à des hypothèses antérieurement proposées, l'esclavage n'a pas été un statut contradictoire mais complémentaire de celui de dépendant. Tout au plus peut-on constater que davantage de femmes restaient esclaves, tandis que les esclaves mâles avaient plus de chance d'évoluer vers le statut de client ou dépendant. En définitive, la corrélation apparaît évidente entre la construction interne des États et la mise en place d'une organisation esclavagiste où, quoi qu'en aient cru et dit les ethnologues instruits de façon souvent tendancieuse par les chefs, la captivité et les souffrances des plus démunis ne furent pas moins âpres que celles des autres esclaves de l'histoire.

3 Toutes les études sont intéressantes. La plus originale est sans doute la première, de David Schoenbrun, qui s'intéresse à l'évolution du vocabulaire des langues locales pour désigner l'esclave et l'esclavage. Le sens s'en est figé au XIX siècle avec la prédominance des traites inter- et extra-africaines sous l'influence des circuits arabes et européens. Mais les mots utilisés démontrent la parenté du vocabulaire utilisé depuis le XVI ${ }^{\mathrm{e}}$ siècle pour désigner des concepts voisins, tous liés à la crainte, à la guerre et au pillage, à la perte de l'honneur, à la honte et au mépris, bref à ce qui pouvait dès lors s'appliquer à l'esclavage tel que conçu en ces temps reculés.

4 L'autre étude qui plaide pour une institution ancienne de l'esclavage dans la région est le chapitre de Richard Reid sur la culture de guerre au Buganda du XVII ${ }^{e} a u$ XIX ${ }^{e}$ siècle. Il établit un parallèle chronologique entre l'expansion du royaume et son organisation esclavagiste. on ne pouvait y confondre paysans libres et esclaves, et le travail des esclaves devint le facteur déterminant de l'organisation économique et sociale ganda (p. 147). Or, avant le xix siècle, on ne décèle pas de rapport évident entre l'usage interne des esclaves et la traite internationale : il s'agit bien d'un système de pouvoir régional relativement autochtone, où la guerre était la pourvoyeuse des esclaves utilisés pour la survie locale.

5 Ceci interpelle la conviction de Jean-Pierre Chrétien selon lequel le fait que la traite arabo-swahili n'aurait touché les confins du Burundi et du Rwanda qu'au xix siècle démontrerait que ces petits royaumes auraient été en somme les seuls à ne pas pratiquer l'esclavage. La traite, affirme-t-il, était pratiquée essentiellement par des étrangers. Il cite entre autres un témoignage néanmoins contradictoire de 1916 
affirmant à deux phrases de distance, d'une part qu'au Burundi, « comme au Ruanda, il n'y a pas ou plus d'esclavage", mais d'autre part que les "jolies jeunes filles tutsi étaient achetées très cher pour garnir les harems des Arabes ou de riches Swahili noirs » (p. 227). Il est possible que l'esclavage masculin ait été limité dans ces pays, mais c'est négliger le cas des femmes : Jan Vansina quant à lui estime que l'armée rwandaise capturait déjà femmes et enfants dès le XVII siècle (p. 21). Or il importe de distinguer l'esclavage (qui est un mode d'organisation sociale interne) de la traite négrière, qui est une activité économique marchande internationale.

6 Cette distinction est renforcée par l'étude d'Edward Steinhart sur l'esclavage en Ankole, petit royaume peu étudié proche de ses voisins méridionaux, le Rwanda et le Burundi : comme pour ces derniers, on sait très peu de chose sur l'esclavage pratiqué en Ankole. Mais plutôt que d'en conclure que l'institution n'existait pas, Steinhart cherche à comprendre pourquoi on en a si peu parlé. Il incrimine, entre autres, les déformations subies sur la connaissance du pays du fait des informations rapportées par le premier chercheur sur la question, K. Oberg, dans les années 1930, et ses successeurs - tous se sont fiés aux informations recueillies exclusivement auprès de la catégorie dominante des Bahima, qui se considéraient comme les seuls sujets véritables du royaume. Ils ont globalement présenté les autres habitants, les Bairu ou Banyankole, qui constituaient la grande majorité de la population, comme des cultivateurs dépendants assimilés à des serfs plutôt qu'à des esclaves. Cette façon de focaliser sur les relations entre Bahima et Bairu (comme dans les États voisins entre Tutsi et Hutu) a durablement empoisonné les analyses portant sur la politique et la société ankole. Le résultat fut qu'on a négligé tout examen sérieux de la différentiation sociale au sein même des Bairu, omettant d'affiner les formes d'oppression sociale entre riches et pauvres, exploiteurs et exploités : on n'a pas posé la question de l'esclavage; or si les esclaves des Bahima étaient tous des Bairu, tous les Bairu n'étaient pas esclaves, ceux-ci victimes en somme d'une sorte de «mort sociale ».

7 Même si les esclaves semblent effectivement avoir joué moins qu'ailleurs, et notamment moins qu'en Ouganda un rôle dans la formation de l'État, les caractéristiques de l'esclavage se retrouvent dans la société précoloniale dans quatre traits attribués par les informateurs à ceux qu'ils désignent du mot d'aba-shumba: le fait d'effectuer un travail non rémunéré, la coercition, le pouvoir du chef de famille de disposer de leur personne pour les donner, pour payer une dette, ou pour en céder la propriété à titre définitif, bref d'en disposer comme d'une tête de bétail ; ce qui faisait de l'esclave un esclave était moins les formes du travail auquel il était astreint, qui différaient assez peu entre domestiques, dépendants, femmes et esclaves, que l'état de honte («being without honour») qui leur était imposé, que ce soit dans les rapports sociaux, affectifs ou sexuels. La caractéristique principale est, en définitive, l'humiliation et la dégradation liées à leur statut éminemment méprisable, qui continue encore aujourd'hui d'empoisonner les rapports sociaux dans l'Ankole moderne. On retrouve en somme le même constat que celui posé dans l'histoire du vocabulaire effectuée par David Schoenbrun.

8 Il existe aussi un rapport entre l'histoire de l'esclavage et l'histoire démographique, comme il a déjà été démontré ailleurs chez les populations lagunaires d'Afrique occidentale ${ }^{2}$. Shane Doyle consacre son essai à l'étude de l'interrelation au Bunyoro entre l'évolution de l'esclavage, de la fécondité et du lignage : bien que son étude porte essentiellement sur le $\mathrm{xIX}^{\mathrm{e}}$ siècle, il insiste sur l'ancienneté et la prospérité d'un 
royaume, le plus peuplé de la région, qui tirait partie de sa force de son organisation esclavagiste - car l'auteur n'agrée pas à l'hypothèse de Claude Meillassoux suivant lequel être esclave aurait eu sur la fécondité des femmes un effet négatif. Si on le comprend bien, ce qui affaiblit le Bunyoro, ce n'est pas l'organisation interne de l'esclavage qui paraît avoir été ancienne, et être devenue vers le milieu du XIx $x^{e}$ siècle une pratique généralisée. Ce qui change la donne, c'est son entrée dans les circuits interafricains de la traite des esclaves, impliquant migrations forcées, interférant avec les politiques matrimoniales et réduisant l'espérance de vie. La prospérité tardive du Bunyoro, son adoption massive des fusils de traite, sa résistance à l'impérialisme égyptien et sa réputation de bastion de la traite négrière en firent pour les Britanniques le pouvoir à abattre. Vers 1899 , le trafic négrier qui avait fait sa force engendra une crise démographique majeure qui le conduisit à la catastrophe.

Deux autres études du volume, également focalisées sur la seconde moitié du XIXe siècle où les sources, écrites autant qu'orales, commencent à abonder analysent les transformations internes du statut d'esclave liées à l'intensification des traites internationales, d'une part en pays unyamwezi entre 1860 et 1900 (Jan-Georg Deutsch), et d'autre part dans le Congo oriental entre 1850 et 1910 (David Northrup). En pays nyamwezi, des témoignages estiment qu'à la fin du XIX siècle les trois quarts de la population étaient des esclaves. L'activité internationale des jeunes hommes du pays, de plus en plus impliqués dans le commerce caravanier avec la côte, a exigé la mise en place autochtone d'une activité productrice importante notamment en vivres. Les Nyamwezi ont utilisé, pour ce faire, des travailleurs étrangers (entre autres burundais) le plus souvent esclaves. L'utilisation interne des esclaves fut aussi favorisée par les entraves mises par les Britanniques à la traite internationale dans l'océan Indien. Au total, le pays nyamwezi fut toujours davantage une zone importatrice qu'exportatrice d'esclaves. Bref il s'est produit au XIXe siècle un processus comparable à celui décrit par Claude Meillassoux dans les États djihadistes d'Afrique de l'Ouest : la mise en place d'un « mode de production esclavagiste » à l'africaine.

10 Quant au Congo oriental, de façon plus classique, Northrup y constate l'utilisation de l'esclavage local par les premiers agents du roi Léopold par le biais du "rachat" d'esclaves en vue de les transformer en travailleurs forcés. Enfin, sur le cas des soldats nubiens du Nil occidental enrôlés par Idi Amin Dada, Mark Leopold s'attache à montrer les scories contemporaines léguées par le passé esclavagiste de la région. Ce passif actuel constitue un volet tout à fait important de l'histoire de l'esclavage.

11 Ce qui manque à ce recueil qui se consacre à l'esclavage comme statut social bien davantage qu'à la traite ou commerce des esclaves (à l'exception de Jean-Pierre Chrétien), c'est une conclusion générale qui pourrait résumer les thèses proposées de la façon suivante: 1 . L'esclavage est attesté depuis très longtemps dans les sociétés de la région, qu'il fût indissociable de la formation même des États considérés (comme au Buganda ou en pays nyamwezi), ou qu'il se contente de coexister avec d'autres formes de dépendance (comme dans d'autres petits États interlacustres). 2. En ce dernier cas, il n'y avait pas contradiction mais complémentarité entre le clientélisme et l'esclavage, dans des formations sociales dont il ne faut pas minimiser la complexité sociale interne. 3. Les femmes étaient particulièrement soumises à l'esclavage, et ce trait s'est fortement accru partout au XIX ${ }^{e}$ siècle. 4. Ce n'est qu'au XIX ${ }^{e}$ siècle, incontestablement, que le statut d'esclave s'est généralisé, compte tenu de la pression exercée par les courants internationaux de la traite qui ont traversé de relais en relais l'Afrique de part 
en part. Mais à l'exception de certaines d'entre elles (au premier chef du Buganda et du Bunyoro, voire des Nyamwezi), les formations locales, compte tenu de leur éloignement des marchés, n'ont pas ou peu participé directement au trafic négrier. C'est ce qui leur a permis d'échapper à la vindicte immédiate des conquérants européens. Néanmoins, compte tenu du contexte global, c'est de façon interne que les sociétés précoloniales ont alors démultiplié en leur sein la condition servile.

Chaque article est nourri de références précises nombreuses, à la façon érudite des travaux de langue anglaise. En outre, l'ouvrage inclut une bibliographie générale abondante (pp. 252-267) presque exclusivement de langue anglaise. Bien entendu, compte tenu de la faiblesse des sources antérieures au $\mathrm{XIX}^{\mathrm{e}}$ siècle, les hypothèses proposées, toutes convaincantes qu'elles paraissent, restent sujettes à discussion. Néanmoins, le tout fait de ce travail un modèle d'érudition cohérente, bref un instrument de travail incontournable pour quiconque s'intéresse soit à la région soit, plus généralement, à l'histoire de l'esclavage africain et en Afrique.

\section{NOTES}

1. Henri MÉDARD, «In Introduction ", in H. MÉDARD \& S. D OYLE (eds.), Slavery in the Great Lakes Region of East Africa, Oxford, James Currey, 2007, p. 2.

2. Harris MÉmeL-Fote, L'esclavage dans les sociétés lignagères de la forêt ivoirienne (XVII Paris, IRD ; Abidjan, Les Éditions du CERAP, 2007. 